\title{
Alpha coma in an adolescent with diabetic ketoacidosis
}

\author{
Slavica Ostojic ${ }^{1}$, Rade Vukovic ${ }^{2}$, Tatjana Milenkovic ${ }^{2}$, Katarina Mitrovic ${ }^{2}$, Milena Djuric ${ }^{1,3}$, \\ Ljubica Nikolic ${ }^{4}$ \\ ${ }^{1}$ Department of Neurology, ${ }^{2}$ Department of Endocrinology, ${ }^{4}$ Intensive Care Unit, Mother and Child Health Care Institute \\ of Serbia "Dr. Vukan Cupic", Belgrade, Serbia; ${ }^{3}$ School of Medicine, University of Belgrade, Serbia. \\ E-mail: radevukovic9@gmail.com \\ Received: 28th December 2016, Accepted: 5th May 2017
}

SUMMARY: Ostojic S, Vukovic R, Milenkovic T, Mitrovic K, Djuric M, Nikolic L. Alpha coma in an adolescent with diabetic ketoacidosis. Turk J Pediatr 2017; 59: 318-321.

This is the first report of alpha coma (AC) caused by brain edema in a patient with diabetic ketoacidosis (DKA). A previously healthy 15-year-old girl was admitted to the intensive care unit due to altered state of consciousness during the course of treatment for DKA. Patient was in a coma, intubated and had tachycardia with poor peripheral perfusion. Results of laboratory analyses indicated severe DKA and computed tomography scan indicated diffuse brain edema. The EEG pattern showed uniform alpha activity. Treatment with intravenous fluids, insulin and mannitol was started. Patient's state of consciousness gradually improved and on the third day she was extubated. On the fifth day, her neurologic status and EEG findings were completely normal with no residual neurological deficits. In conclusion, although AC is associated with a high fatality rate, favorable outcome can be achieved with prompt recognition and treatment of cerebral edema in pediatric patients with DKA.

Key words: alpha coma, brain edema, diabetic ketoacidosis, type 1 diabetes mellitus, outcome.

Brain edema is a rare, potentially fatal complication of diabetic ketoacidosis (DKA) which occurs in up to $1 \%$ of patients with DKA $^{1-4}$. Neurological deterioration after the initial period of clinical improvement is an ominous sign suggesting the development of brain edema1,3. This severe complication of DKA is more frequently seen in younger patients, with newly diagnosed diabetes and longer duration of symptoms, as well as in patients with low socio-economic status ${ }^{1,4,5}$. Having that in mind, brain edema in DKA represents primarily as an issue in the pediatric population. Rate of mortality in children with brain edema in DKA is very high, $21-25 \%$ according to available data ${ }^{2,4-5}$.

Clinical assessment of comatose patients relies heavily on neurophysiological procedures, such as electroencephalography (EEG) and evoked potentials (EP), and these procedures indicate the degree of brain dysfunction, aiding in the assessment of type of brain impairment, depth of coma and clinical follow up of these patients ${ }^{6}$. Rhythmic patterns of alpha, beta, theta and spindle frequencies in comatose patients determine the coma type as alpha, beta, theta and spindle coma ${ }^{6}$. Alpha coma (AC) is mainly seen in comatose patients with brainstem damage, anoxic encephalopathy, after cardiopulmonary arrest and in toxic encephalopathies caused by drugs or metabolic disorders ${ }^{6,7}$. Alpha coma associated with DKA is a rare finding, and to our knowledge, there has only been one published case of AC in a pediatric patient with DKA, caused by hemorrhagic infarction of the brain ${ }^{7}$. Survival rates and long-term neurological outcomes of patients in AC vary widely depending on the etiology of $\mathrm{coma}^{6,8}$.

This case report presents clinical course and outcome of AC caused by brain edema during DKA in a 15-year-old adolescent girl. 


\section{Case Report}

A previously healthy 15-year-old adolescent girl was admitted to the intensive care unit (ICU) of our clinic due to the development of coma during treatment for DKA. Her symptoms began one month before the admission and consisted of classical constellation of polydipsia, polyuria, polyphagia and weight loss. Three days prior to the admission, she complained about abdominal pain, nausea and diarrhea. On the day of the admission, she was examined in a regional hospital. Prior to any procedures performed, informed consent was obtained. She was noted to be of ill general appearance, drowsy, dehydrated, with cold extremities, tachycardic (140 bpm), with Kussmaul's breathing and her breath smelled of ketones. Her Glasgow comma score (GCS) was 14. Results of laboratory investigations indicated severe diabetic ketoacidosis (glucose $36 \mathrm{mmol} / \mathrm{L}$, $\mathrm{pH} 6.8$, serum bicarbonate $5.7 \mathrm{mmol} / \mathrm{L}$ ). Although the treatment of diabetic ketoacidosis was promptly started, the clinical condition continued to deteriorate, with vomiting of dark brown gastric content and a rapid decline in the level of consciousness from somnolence to stupor. She was intubated and transported to our clinic. Total intravenous fluid intake was $1800 \mathrm{ml}$ during the first 12 hours and $300 \mathrm{ml}$ $\mathrm{NaCl} 0.9 \%$ during the 2.5 hours of transport.

At the admission to our clinic she was in a coma, with a GCS of 6 . She was intubated,

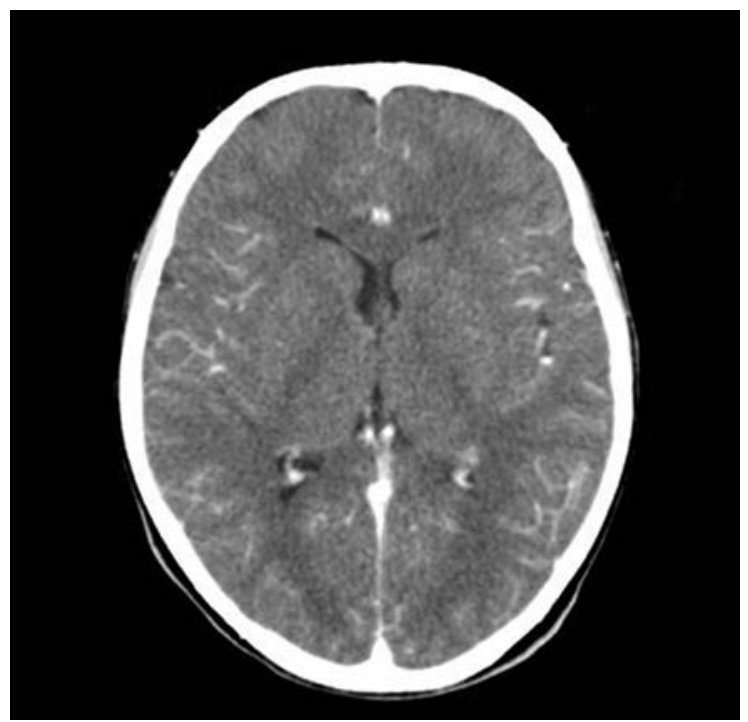

Fig. 1. Head CT scan on admission showing diffuse brain edema tachycardic (171 bpm), normotensive (116/73 $\mathrm{mmHg}$ ), with cold periphery, pale skin and visible signs of dehydration. Results of laboratory analyses were consistent with severe DKA: glucose $20.5 \mathrm{mmol} / \mathrm{L}, \mathrm{pH} 6.9, \mathrm{pCO}_{2}$ $14 \mathrm{mmol} / \mathrm{L}$, serum bicarbonate $4.0 \mathrm{mmol} / \mathrm{L}$, HbA1c $16 \%$ with positive urine ketones. C-reactive protein (CRP) was $221 \mathrm{mg} / \mathrm{L}$ and electrolytes, BUN, creatinine and liver function tests were within normal limits. Computed tomography (CT) of the brain showed diffuse brain edema (Fig. 1), and EEG showed uniform alpha activity without specific discharges and no response to external sensory stimuli (Fig. 2A).

In the management of cerebral edema, $20 \%$ mannitol was administered every six hours in a dose of $0.5 \mathrm{mg} / \mathrm{kg}$ and she was ventilated using synchronized intermittent mandatory ventilation with carbon dioxide partial pressure $\left(\mathrm{pCO}_{2}\right)$ maintained in the range of $25-30 \mathrm{mmHg}$ during the next two days. DKA was managed according to the appropriate protocols, with continuous infusion of regular insulin in the dose of $0.15 \mathrm{IU} / \mathrm{kg} / \mathrm{h}$. Dark brown content was noted in the nasogastric tube and treatment

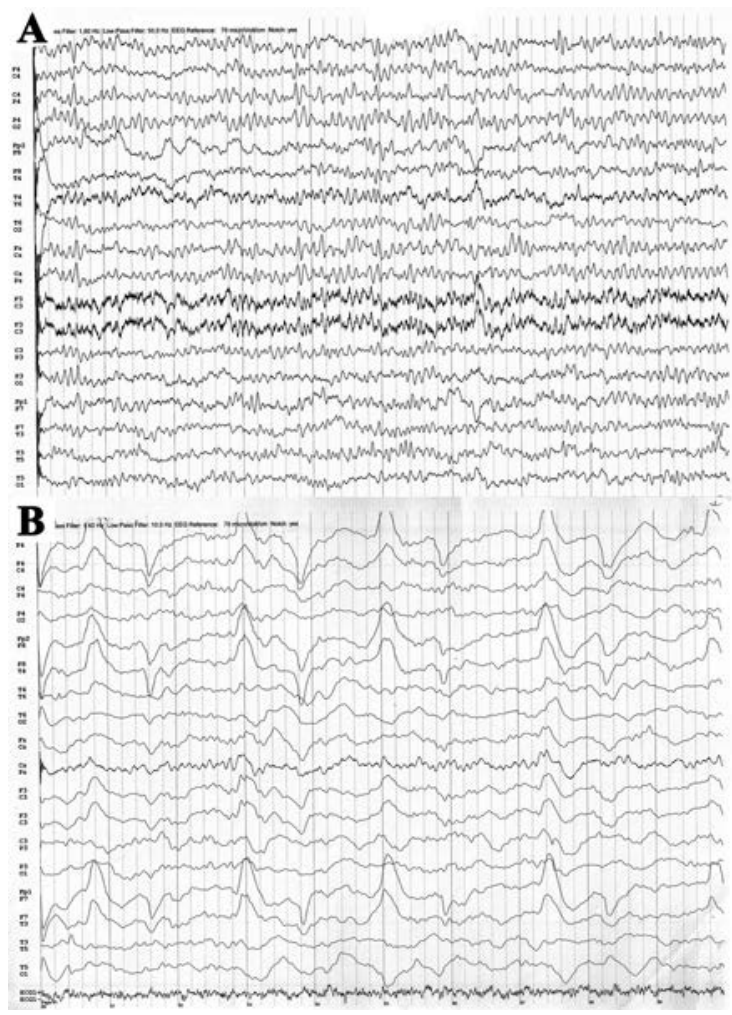

Fig. 2. A) EEG recorded on admission showing uniform alpha activity, B) EEG recorded 24 hours after admission showing high voltage theta and delta waves 
with pantoprazole was started due to suspected stress ulcer. Antibiotic therapy with ceftriaxone was administered for possible systemic infection and nasogastric suctioning was started due to development of paralytic ileus caused by hypokalemia.

Acidosis was gradually corrected during the first 24 hours of treatment, but no improvement in the state of consciousness could be registered during the first day, with GCS ranging from 6 to 8 . On the second day EEG showed slowing of background activity with the appearance of high voltage theta and delta waves and slightly better reactivity to external stimuli (Fig. 2B). 48 hours after admission a gradual improvement in the level of consciousness was observed, with an increase of GCS to 11. During the third day of hospitalization, neurological improvement continued, patient stopped vomiting, she was extubated and started on oral intake. The administration of insulin was continued by subcutaneous injections. On the fifth day, her neurologic status and EEG findings were completely normal with no residual neurological deficits. Further treatment was continued in the department of endocrinology with multiple daily injections of insulin.

\section{Discussion}

Brain edema is the main cause of mortality in pediatric patients with $\mathrm{DKA}^{3}$. It is presumed that pathophysiology of brain edema in DKA is more likely of vasogenic than cellular nature, caused by blood-brain barrier disruption with increased vascular permeability and brain inflammatory response ${ }^{9,10}$. Although the very treatment of DKA is considered to be the main culprit for the development of brain edema, the nature of the pathophysiological processes in DKA is accountable for neurological complications of this condition: accumulation of intracellular osmoles, elevated levels of vasopressin, the increase in the concentration of atrial natriuretic factor 24 hours after the start of therapy, hypoxia and ischemia, abnormality in numbers and functions of aquaporin channels ${ }^{10}$. The most important risk factors for the development of brain edema in DKA are: rapid correction of electrolyte disturbances, application of bicarbonates, intensive hyperventilation (resulting in too low $\mathrm{pCO}_{2}$ ) and a younger age 10,11 . Brain edema is the most serious complication of DKA, with a high rate of mortality (21-25\%) and morbidity (30-40\%), so understanding pathophysiology, prevention and treatment of cerebral edema is paramount in improving the outcome of $\mathrm{DKA}^{2,4}$.

Continuous monitoring of vital functions and frequent assessment of neurologic status are essential for the early recognition of these complications. An evidence-based protocol for bedside neurological evaluation has been proposed by Muir et al. ${ }^{12}$ for early recognition of neurological complications of DKA: major criteria (altered level of consciousness, bradycardia or age-inappropriate incontinence) and minor criteria (vomiting, headache, lethargy and hypertension). Our patient met the clinical criteria for brain edema which was confirmed with head CT scan. She initially developed headache, vomiting and lethargy, which were followed by deterioration into a deep coma.

The finding that separates the presented patient from other patients with brain edema in DKA was the fact that she was in a state of AC. Alpha activity indicates the rhythm of $8-13 \mathrm{~Hz}$ in the waking state, with maximum amplitude of waves over the occipital region of the brain. In the AC, alpha waves are distributed uniformly both anteriorly and posteriorly regions of the brain with mild frontal predominance ${ }^{13}$. Unlike normal rhythms, these waves appear without appropriate accompanied waveforms, with pathological topographic pattern and show no normal reactivity to external sensory stimulus, as was the case in our patient. In most comatose patients, AC is a transient EEG pattern which eventually changes to Theta or Delta coma ${ }^{14}$. The prognosis for most patients in AC is usually poor, depending on the etiology of the coma. When the etiology of AC is cardiorespiratory arrest or other anoxic brain injury, the mortality is 8.5 times higher compared to other etiologies, including metabolic causes ${ }^{15}$. In our patient, EEG pattern changed, showing slowdown in background activities with the advent highvoltage slow Theta and Delta waves. In this case, alpha rhythm was unreactive to external stimuli during the first day, but it had become reactive on the second day of hospitalization. This is considered to be a good prognostic sign. On the fifth day of treatment, the EEG findings returned to normal, which was followed by apparent clinical improvement. Repeated EEG 
monitoring with careful clinical assessment showed to be of great importance in the treatment of our patient.

To our knowledge, this is the first case report of AC caused by brain edema in a patient with DKA. Shoar et al. ${ }^{7}$ reported AC in a female adolescent with DKA, although caused by intraparenchymal hemorrhage, whose outcome was also favorable, without any neurological impairment and sequelae ${ }^{7}$. Together, our findings point out that although AC is generally associated with a poor prognosis and high fatality rate, the outcome depends on etiology and preexisting state of the patient's health and can be favorable in pediatric patients with DKA.

The proper treatment of ketoacidosis and cerebral edema in intensive care units provide an opportunity for a positive outcome both in terms of survival, and long-term neurological outcome. However, mortality associated with brain edema is high, and the best way to prevent brain edema in DKA is the prevention of ketoacidosis itself using nationwide preventive public-health measures ${ }^{1}$. Patients with DKA should be carefully monitored with repeated assessments of neurological state in order to recognize early symptoms and signs of impending brain edema.

\section{Acknowledgments}

The authors would like to express sincere appreciation to all the doctors and nurses of the Intensive Care Unit of Mother and Child Health Care Institute that were involved in the treatment of this patient.

\section{REFERENCES}

1. Watts W, Edge JA. How can cerebral edema during treatment of diabetic ketoacidosis be avoided? Pediatric Diabetes 2014; 15: 271-276.

2. Wolfsdorf J, Glaser N, Sperling MA. Diabetic ketoacidosis in infants, children, and adolescents: a consensus statement from the American Diabetes Association. Diabetes Care 2006; 29: 1150-1159.

3. Wolfsdorf JI. The International Society of Pediatric and Adolescent Diabetes guidelines for management of diabetic ketoacidosis: do the guidelines need to be modified? Pediatric Diabetes 2014; 15: 277-286.

4. Edge JA, Hawkins MM, Winter DL, Dunger DB. The risk and outcome of cerebral oedema developing during diabetic ketoacidosis. Arch Dis Child 2001; 85: 16-22.

5. Glaser N, Barnett P, McCaslin I, et al. Risk factors for cerebral edema in children with diabetic ketoacidosis. N Engl J Med 2001; 344: 264-269.
6. Sutter R, Kaplan PW. Electroencephalographic patterns in coma: when things slow down. Epileptologie 2012; 29: 201-209.

7. Shoar Z, Dinne C, Yorns W, De Luca F, Rezvani G. Diabetic ketoacidosis with cerebral hemorrhage and alpha coma in an adolescent female. J Peditr Endocrinol Metab 2013; 26: 561-564.

8. Iragui VJ, McCutchen CB. Physiologic and prognostic significance of "alpha coma". J Neurol Neurosurg Psychiatry 1983; 46: 632-638.

9. Rasker RC, Acerini CL. Cerebral edema in children with diabetic ketoacidosis: vasogenic rather than cellular? Pediatr Diabetes 2014; 15: 261-270.

10. Levin DL. Cerebral edema in diabetic ketoacidosis. Pediatr Crit Care Med 2008; 9: 320-329.

11. Wolfsdorf JI, Allgrove J, Craig ME, et al. ISPAD Clinical Practice Consensus Guidelines 2014 Compendium. Diabetic ketoacidosis and hyperglycemic hyperosmolar state. Pediatr Diabetes 2014; 15: 154-179.

12. Muir AB, Quisling RG, Yang MC, Rosenbloom AL. Cerebral edema in childhood diabetic ketoacidosis: natural history, radiographic findings, and early identification. Diabetes Care 2004; 27: 1541-1546.

13. Westmoreland BF, Klass DW, Sharabrough FW, Reagan TJ. Alpha-coma. Electroencephalographic, clinical, pathologic, and etiologic correlations. Arch Neurol 1975; 32: 713-718.

14. RamachandranNair R, Sharma R, Weiss SK, Otsubo H, Cortez MA. A reappraisal of rhythmic coma patterns in children. Can J Neurol Sci 2005; 32: 518-523.

15. Kaplan PW, Genoud D, Ho TW, Jallon P. Etiology, neurologic correlations, and prognosis in alpha coma. Clin Neurophysiol 1999; 110: 205-213. 